\title{
DÉTECTION À HAUTE RÉSOLUTION SPATIALE DE LA DESSERTE FORESTIĖRE EN MILIEU MONTAGNEUX
}

\author{
António Ferraz ${ }^{1,2,3}$, Clément Mallet ${ }^{1}$, Nesrine Chehata ${ }^{1,4}$ \\ 1: Université Paris-Est, IGN, SRIG, MATIS, France \\ prenom.nom@ign.fr \\ 2 : R\&D Unit INESC Coimbra, Portugal
}

3 : NASA postdoctoral program fellow, NASA - Jet Propulsion Laboratory, Etats-Unis

4: Bordeaux INP, EA 4592, F-33607 Pessac, France

\begin{abstract}
Résumé
En milieu montagneux et forestier, la localisation de la route et ses caractéristiques géométriques sont des informations cruciale pour de nombreuses applications écologiques et liées à la gestion forestière. Par ailleurs, le lidar aéroporté topographique est devenu une technique de télédétection reconnue pour la caractérisation fine de la surface terrestre : les Modèles Numériques de Terrain (MNT) en sont le produit standard.

Cet article aborde le problème de la détection de routes sur de grandes surfaces $\left(>1000 \mathrm{~km}^{2}\right)$ dans de tels environnements. Pour cela, nous avons proposé une méthode fondée sur l'hypothèse que les routes peuvent être modélisées par des objets planaires suivant une direction privilégiée et avec de fortes variations du relief dans la direction orthogonale. La connaissance seule du MNT lidar à $1 \mathrm{~m}$ de résolution est suffisante dans notre processus, qui ne requiert donc pas le traitement supplémentaire des nuages de points 3D lidar ni de données à retour d'onde complète. L'intégralité de l'analyse se fait donc en deux dimensions. Tout d'abord, trois attributs morphologiques sont extraits du MNT et introduits dans une classification supervisée par Forêts Aléatoires des zones potentiellement "routes". Ensuite, un graphe est créé à partir de ce masque de focalisation afin de combler les éventuels manques et occlusions dus principalement à la végétation. En particulier, les nœuds sont sélectionnés avec un Processus Ponctuel, puis le graphe est élagué en suivant le modèle de route initial. Enfin, la largeur et la pente des routes sont estimées grâce au MNT avec une analyse orientée-objet. D'une part, on obtient une qualité de détection convaincante, tant au niveau de l'exhaustivité ( $>80 \%$ ) que de la précision géométrique, supérieure à celle des bases de données topographiques 2D existantes. De plus, de nouvelles routes sont détectées grâce à la capacité du lidar à restituer le terrain sous le couvert végétal. Cependant, en présence d'un trop faible nombre de mesures lidar au niveau du sol, des routes peuvent ne pas être restituées. Enfin, nous montrons que notre méthode est adaptée à une analyse sur de grandes surfaces puisqu'elle permet des rendements de moins de 2 minutes par $\mathrm{km}^{2}$.
\end{abstract}

Mots clés : Lidar aéroporté, Modèle Numérique de Terrain, dessertes, classification, zones montagneuses, forêts, cartographie sur de grandes surfaces.

\begin{abstract}
In forested mountaneous areas, the road location and its geometric description are necessary information for many purposes linked to ecological issues. Topographic airborne laser scanning has become an established technique to characterize the Earth surface. Lidar provides $3 D$ point clouds allowing for a fine reconstruction of the topography : very high resolution Digital Terrain Models (DTM) are the key products.

This paper addresses the problem of road detection and characterization in such environments over large areas $\left(>1000 \mathrm{~km}^{2}\right)$. For that purpose, we assume that roads can be modelled as planar elongated features with relief variation in orthogonal direction. The knowledge of the $1 \mathrm{~m}$ lidar DTM is sufficient and no additional 3D point cloud processing method needs to be involved nor full-waveform data analysis. First, a carefully designed set of morphological features is defined as input for a supervised Random Forest classification of potential road patches. Then, a graph is built over these candidate regions : vertices are selected using a Point Process and edges are created in order to fill gaps created by vegetation occlusion. Such a graph is pruned using morphological criteria derived from the input road model. Finally, road width and slope are retrieved using an object-based approach. Results show that roads are correctly retrieved (>80\%) and with higher planimetric accuracy than $2 D$ existing topographic databases. Furthermore, additional roads can be detected with the ability of lidar sensors to penetrate the understory. However, in case of very dense vegetation and insufficient morphological hints in the DTM, gaps may exist in the results. Eventually, we demonstrate that our method is tailored for large-scale analysis since the full workflow takes less than 2 minutes per $\mathrm{km}^{2}$.
\end{abstract}

Keywords : Lidar, Digital Terrain Model, airborne, road extraction, classification, mountaneous areas, Forests, large scale mapping.

Revue Française de Photogrammétrie et de Télédétection

$n^{\circ}$ 211-212 (décembre 2015) 


\section{Introduction}

\subsection{Problématique}

La connaissance de la localisation, de l'utilisation et de l'état de la desserte forestière est une information cruciale pour de nombreuses applications écologiques et économiques et pour les indicateurs qui en découlent (Lugo et Gucinski, 2000; Robinson et al., 2010; Coffin, 2007). En ce qui concerne la gestion forestière et la biodiversité, il s'agit de trames vertes, de routes pour le déplacement de produits extraits de la forét, d'infrastructure pour les loisirs ou la protection du feu. En milieu montagneux, la fragmentation du paysage peut avoir un impact négatif sur l'écoulement des eaux et, en conséquence sur la qualité de l'eau et des habitats aquatiques ou provoquer des glissements de terrain (Borga et al., 2005; Marcantonio et al., 2013). Pour cela, elles doivent être précisément connues et surveillées, et en conséquence être mises à jour à une fréquence au moins annuelle (Gucinski et al., 2001).

En particulier, l'organisation de la logistique d'approvisionnement en bois-énergie nécessite de connaître les conditions locales de la mobilisation de la ressource : accessibilité par la desserte forestière, difficultés d'exploitation, ainsi que les coûts associés. La caractérisation des conditions de mobilisation de la ressource forestière est le dernier maillon de la chaîne et cette problématique est principalement fondée sur une description fine des réseaux de dessertes en milieux forestiers, sur la valorisation de critères d'exploitabilité intégrant la distance aux dessertes et la pente du terrain, et sur le développement d'indicateurs d'opportunité de récolte (Bellier et al., 2014). Pour cela, il est nécessaire de connaître à la fois la position de la route et ses caractéristiques géométriques (pente, largeur, rayon de courbure) afin de savoir quels types de véhicules pourront emprunter tel ou tel tronçon. Différentes solutions existent pour la collecte et la mise à jour de bases de données (BD) spatiales de la desserte forestière : l'intégration de bases de données topographiques nationales ou régionales, la photo-interprétation, les relevés GPS, méthodes participatives ou portails internet facilitant l'échange de bonnes pratiques, la diffusion de guides méthodologiques et la recherche collaborative et, enfin, la télédétection. Les quatre premières solutions offrent toutes des avantages en terme d'exhaustivité du linéaire routier (Figures 1a-b, sauf parfois sous couvert forestier dense) mais présentent des faiblesses en terme de précision de localisation (surtout pour les relevés GPS) et d'informations attributaires : même dans le cas de BD métiers issues d'initiatives locales, tous les champs d'intérét pour notre problématique peuvent ne pas exister ou ne pas être renseignés (cf. Figures 1a-c). Enfin, la question de la mise à jour de ces informations, quand elles existent, n'est pas résolue.

Dans cet article, nous abordons le problème d'utiliser des données de télédétection, et en particulier des données lidar aéroporté (Vosselman et Maas, 2010) pour la détection et la caractérisation du réseau routier en milieu montagneux et forestier sur de grandes surfaces. Ces dernières offrent de nouvelles perspectives de par leur capacité à pénétrer le couvert végétal, à restituer avec une grande précision altimétrique le terrain en-dessous et par désormais le déploiement de la technologie lidar sur de grandes étendues.

\subsection{Analyse de l'existant}

Un très grand nombre d'articles de la littérature traite de la détection (semi-)automatique des routes mais principalement en milieux urbains et péri-urbains et à partir d'imagerie optique géospatiale (Shackelford et Davis, 2003; Jin et Davis, 2005; Mena et Malpica, 2005; Amo et al., 2006; Mayer et al., 2006). Dans tous les cas, ces méthodes ont été construites pour un environnement et des données spécifiques. Elles se différencient avant tout par le modèle de route sélectionné et qui est lui-même très fortement lié à la résolution spatiale des images.

- Pour des résolutions supérieures à $1 \mathrm{~m}$, les routes sont vues comme des objets fins allongés : des approches globales fondées souvent sur l'extraction de lignes sont privilégiées (Barzohar et Cooper, 1996; Lacoste et al., 2005). Adoptées en milieu péri-urbain, elles permettent de construire directement des réseaux en se ramenant à un problème de graphes (Hinz et Baumgartner, 2003) et de régler les problémes de manque local d'information pertinente (Rochery et al., 2004). Des approches multi-échelles sont souvent adoptées (Laptev et al., 2000) et les détections initiales sont les plus souvent affinées par une étape de suivi de profils (Vosselman et de Knecht, 1995; Hu et al., 2007). Ces méthodes sont d'autant plus pertinentes qu'elles peuvent être intégrées dans des configurations où une connaissance initiale des routes (BD topographique) existe déjà (Fujimura et al., 2008; Miao et al., 2013).

- Pour des résolutions sub-métriques (et les milieux urbains), les routes apparaissent comme des zones homogènes (en géométrie, topologie, texture, couleur) et des approches locales, fondés sur des méthodes de classification, sont sélectionnées (Mena, 2003; Grote et al., 2012; Ziems et al., 2012). Elles permettent d'ajouter des informations contextuelles et de limiter les fausses détections liées aux voitures ou aux passages piétons (Wegner et al., 2013). Elles sont généralement suivies d'une étape de construction de graphe (Unsalan et Sirmacek, 2012; Matkan et al., 2014) puis de vectorisation.

- Enfin, des méthodes couplant raisonnements global et local (Chai et al., 2013; Montoya et al., 2014; Poullis, 2014) sont désormais développées afin de bénéficier des avantages des deux types d'approches.

Les données lidar aéroporté présentent trois avantages 


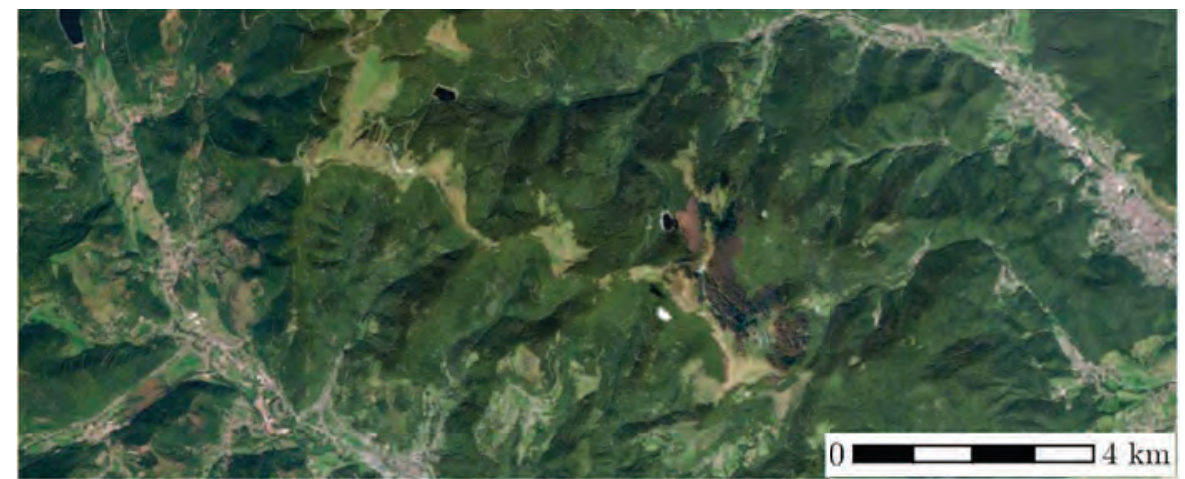

(a)

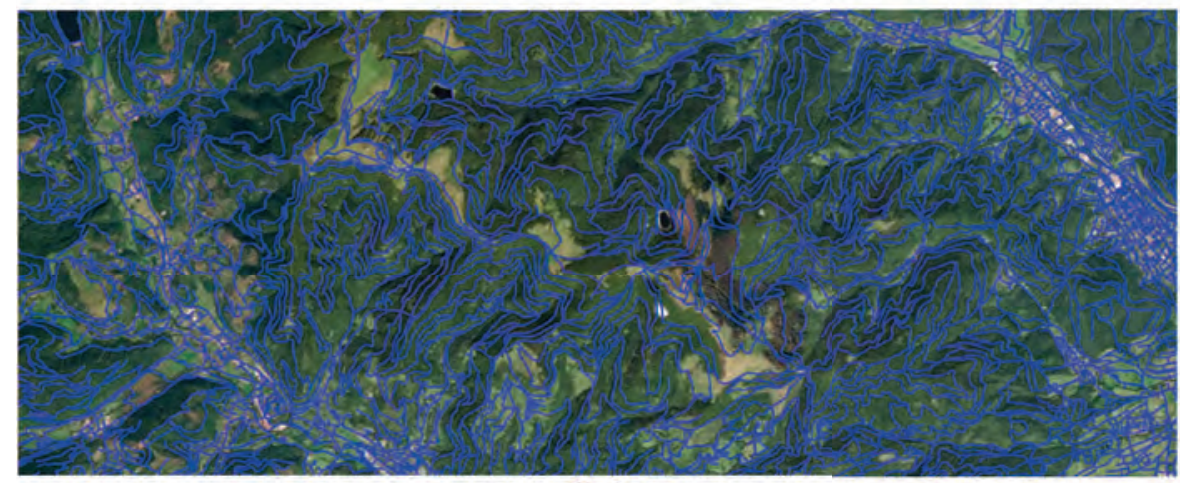

(b)

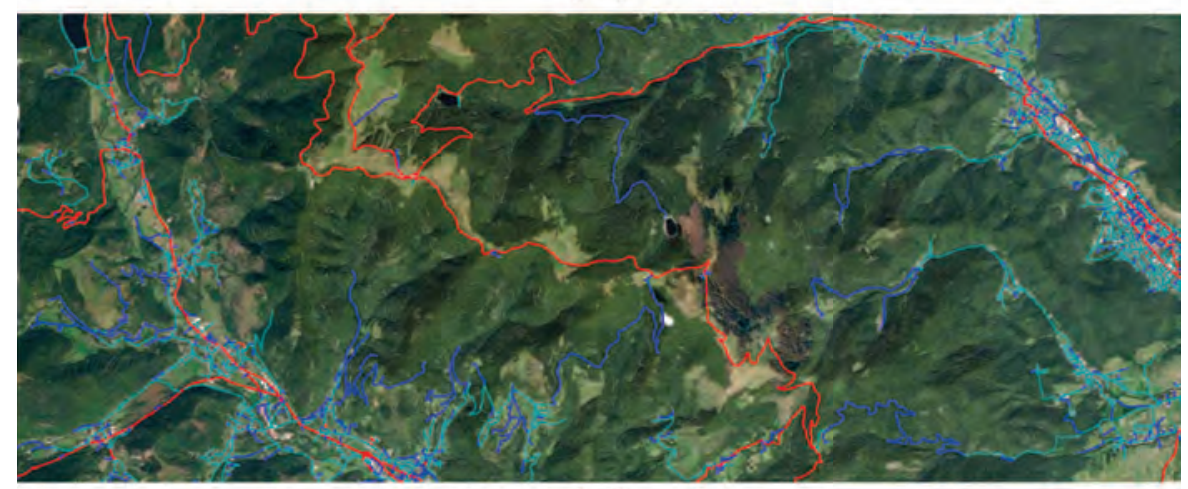

(c)

FIGURE 1 : Pertinence de la détection de la desserte en milieu forestier (a) pour les applications de mise à jour du linéaire routier (b) et de caractérisation géométrique (c). On note souvent que même si le réseau routier est complet dans une base de données topographique, seule une faible proportion des routes a une largeur assignée, et souvent dans des gammes prédéfinies $(\square: 0,1-3 \mathrm{~m} ; \square: 3-6 \mathrm{~m} ; \square 6 \mathrm{~m}$ et plus). 
dans le développement et les performances de ces méthodes. La faculté du faisceau laser à pénétrer sous le couvert forestier permet tout d'abord d'obtenir des Modèles Numériques de Terrain (MNT) à très haute résolution spatiale, sans occlusion, et, en conséquence d'extraire une information d'altitude fiable (Hatger, 2005; Clode et al., 2005; Matkan et al., 2014), des hautes fréquences du relief comme des ruptures de pente et des bords de route (Rieger et al., 1999; White et al., 2010) en zones de pente. Par analyse morphologique des MNT, on accède ainsi à des informations géométriques supplémentaires par rapport aux données optiques standard (Rieger et al., 1999; Poullis et You, 2010) et sans problème d'ombres portées (Hu et al., 2014). Ensuite, l'analyse des nuages de points lidar 3D permet de connaître la répartition verticale des objets au-dessus du sol (Hatger, 2005; David et al., 2009; Poullis et You, 2010; Djuricic et Hollaus, 2013) : en milieu forestier, cela indique en particulier la présence de végétation basse et permet de prédire les zones d'occlusion (Lee et al., 2005). Enfin, l'information d'intensité, souvent dérivée de l'analyse de l'onde lidar (Djuricic et Hollaus, 2013), apporte une information radiométrique absente jusque là et aide à la discrimination des surfaces (routes/bâtiments dans (Clode et al., 2007) et routes/terrain nu dans (David et al., 2009; Azizi et al., 2014)). Toutefois, les techniques de classification fondées sur cette information ne peuvent être facilement transférées aux milieux forestiers car l'on ne sait pas encore la calibrer correctement et la corriger de l'influence du couvert forestier.

\subsection{Solution proposée et contributions}

Nous cherchons à développer une approche qui soit capable de détecter de manière rapide, fiable et sur de grandes surfaces les routes en milieu forestier et montagneux. Les principales méthodes récentes existantes (David et al., 2009; White et al., 2010; Djuricic et Hollaus, 2013) ne sont pas compatibles avec ces exigences puisqu'elles n'ont pas été construites pour des analyses sur de grandes superficies et/ou requièrent des données trop complexes (à savoir des données à retour d'onde complète). Nous proposons une méthode de détection qui ne se fonde que sur des MNT de résolution métrique. Nous ne cherchons pas à restituer un réseau en sortie de processus car, d'une part, il est difficile d'intégrer des connaissances a priori en zones de montagne, et d'autre part, cette étape peut être résolue par des approches proposées dans la littérature et mentionnées ci-dessus. Notre méthode présente plusieurs contributions intéressantes :

- Une méthode de détection automatique et sans paramètres à régler d'une zone à une autre. Elle ne requiert en entrée qu'un faible nombre de pixels routes/non-routes pour la classification supervisée sous-jacente.

- Une approche fonctionnant sur de grandes surfaces qui ne nécessite qu'un MNT en entrée et qui peut être donc reproduite facilement sur d'autres zones et avec d'autres sources de données.

- Une méthode modulaire impliquant des outils libres et qui peut être optimisée à façon : Sherwood (Criminisi et Shotton, 2013) pour la classification supervisée, LibRJMCMC (Brédif et Tournaire, 2012), Orfeo ToolBox (Inglada et Christophe, 2009) et CGAL pour la construction et l'élagage du graphe sous-jacent à la caractérisation géométrique de la desserte.

\section{Méthodologie}

\subsection{Stratégie générale}

Notre stratégie de détection et de caractérisation de la desserte ne se fonde que sur l'analyse en deux dimensions du Modèle Numérique de Terrain lidar à $1 \mathrm{~m}$ de résolution afin de proposer une méthode efficace sur de grandes surfaces. Nous aurions pu intégrer des traitements sur le nuage de points 3D lidar et gagner en qualité de détection et en précision de caractérisation mais au détriment des temps de calcul, vu le volume de données brute à disposition (près de 5,6 milliards de points 3D sur notre zone, cf. Partie 3). Clode et al. (2007) et David et al. (2009) utilisent en particulier l'intensité des échos lidar pour discriminer les zones de routes mais même si cette information est désormais présente dans la grande majorité des nuages de points 3D lidar, (i) on ne peut être assuré d'une bonne calibration de cet attribut sur de grandes zones et (ii) on ne sait pas encore le corriger de l'influence de la canopée en cas d'occlusion de la desserte.

Notre méthode se fonde sur un modèle de route suivant deux hypothèses (Gucinski et al., 2001; Djuricic et Hollaus, 2013) :

- Une route est un objet suivant une direction privilégiée, avec de fortes variations altimétriques dans la direction orthogonale;

- Une route fait entre 2 et $12 \mathrm{~m}$ de largeur.

Notre méthode permet de retrouver les objets en zones de pente mais pas sur les terrains plats. Les processus développés ne sont donc pas applicables dans ces environnements là. La méthode se décompose en trois parties principales, décrites ci-dessous (cf. Figures 2 et 3$)$ :

1. Focalisation : trois attributs sont extraits du MNT et intégrés dans une classification binaire supervisée par Forêts Aléatoires pour obtenir un masque de focalisation sur les routes à la résolution du MNT (1 m).

2. Construction du graphe de route : un graphe de route exhaustif est créé afin de proposer un grand nombre de chemins possibles, puis est élagué seIon les critères géométriques définis ci-dessus.

3. Caractérisation géométrique 3D : la largeur et la pente des tronçons de route sont obtenues, toujours sur le MNT, par une analyse par tronçon, par profil et orientée-objet. 


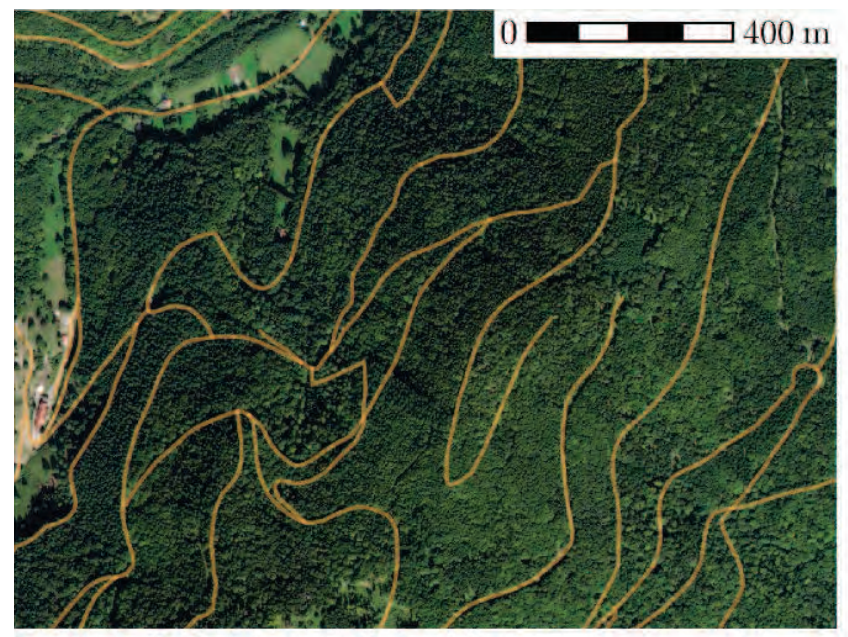

(a)

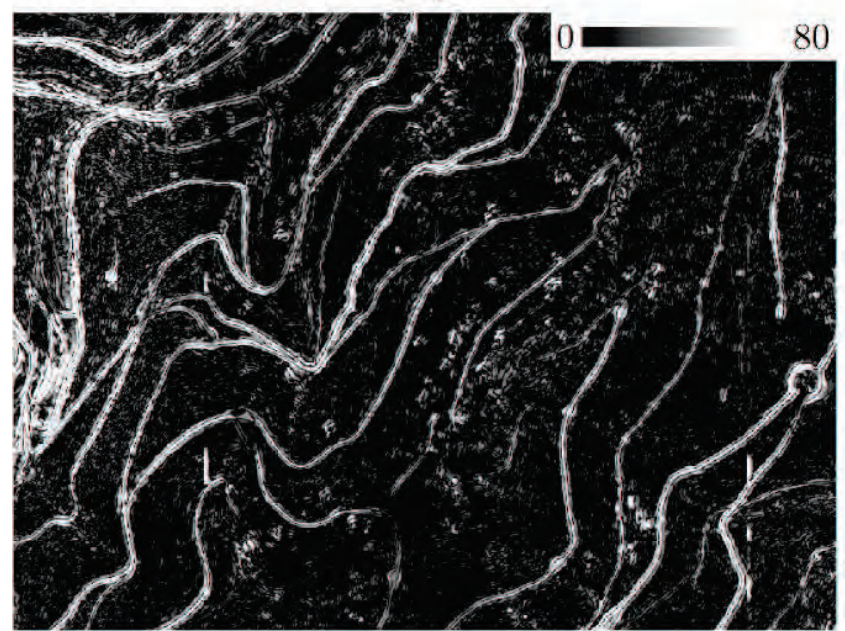

(c)

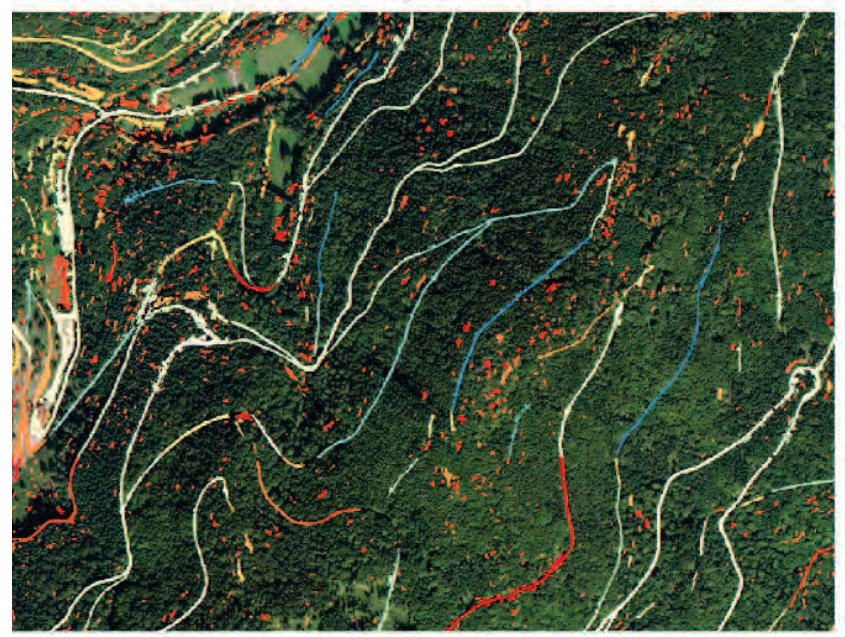

(e)

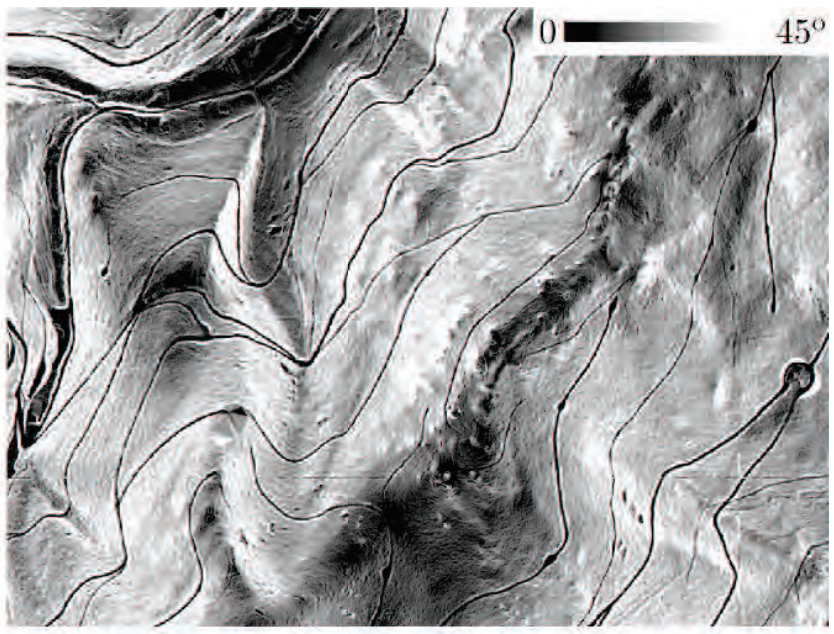

(b)

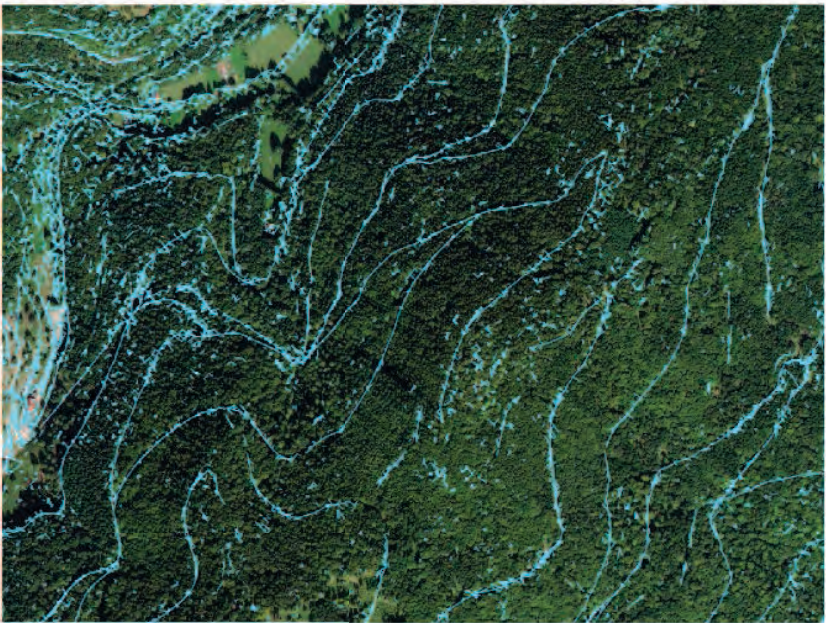

(d)

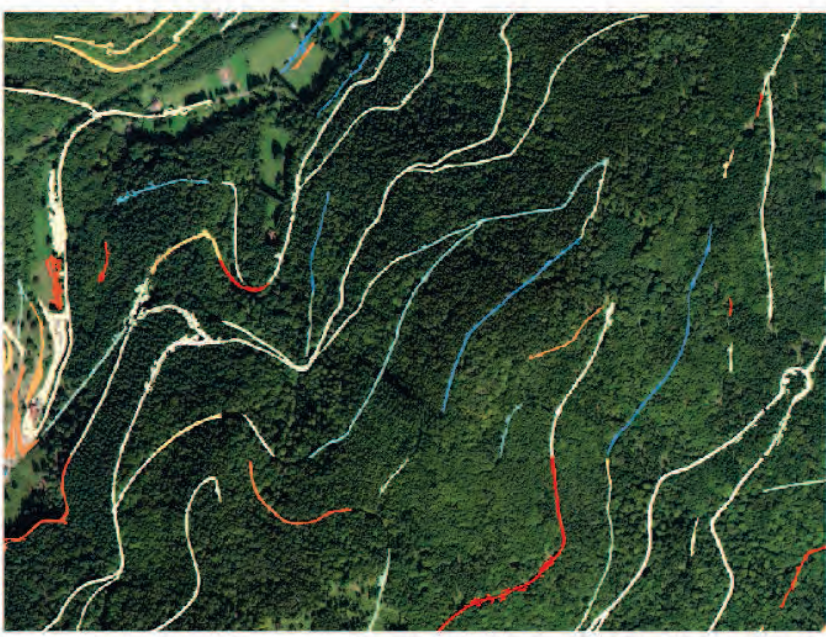

(f)

FIGURE 3 : Illustration de la méthode sur une zone d'intérêt. (a) Orthoimage avec une BD existante. (b) Carte de pente. (c) Carte de pente multi-échelles. (d) Graphe de route après élagage. (e-f) OBIA avant et après filtrage. Chaque segment est coloré selon sa largeur calculée par SFS (cf. Partie 2.4). $\square:<3,5 \mathrm{~m}-\square: 3,5-4,5 \mathrm{~m}-\quad:$ : 4,5-5,5 m- $-: 5,5-6,5 \mathrm{~m}-\square:>6,5 \mathrm{~m}$. 


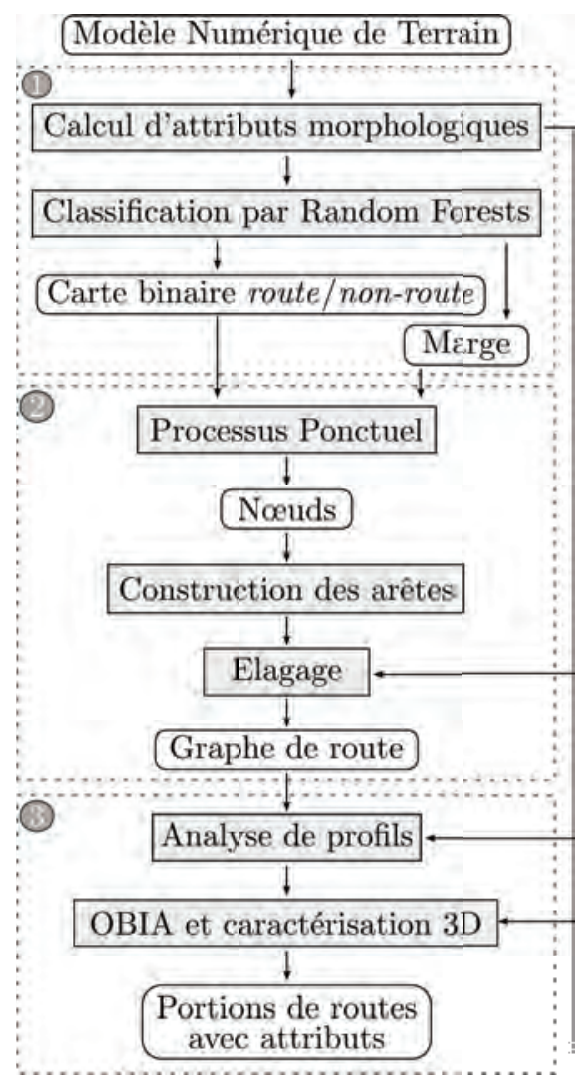

FIGURE 2 : Aperçu général de l'approche proposée. Voir le texte pour plus de détails sur les trois étapes.

\subsection{Focalisation}

\section{Extraction d'attributs}

Trois attributs sont calculés à partir du MNT $M$ à $1 \mathrm{~m}$ : la pente, la rugosité et le gradient de pente. Trois images sont générées à la même résolution et les calculs se fondent sur un voisinage en 8 -connexité. La pente $\mathcal{P}$ est obtenue ainsi :

$$
\forall s, \quad \mathcal{P}(s)=\arctan \left(\sqrt{d X(s)^{2}+d Y(s)^{2}}\right),
$$

oé $d X$ et $d Y$ sont les dérivées de l'altitude respectivement dans les directions Est-Ouest et Nord-Sud autour du pixel $s$. La pente est utilisée afin de détecter les zones de terrain localement plates (Figures 3a-b). La rugosité $\mathcal{R}$ est ensuite calculée pour accentuer les ruptures du relief et mesure l'hétérogénéité du terrain autour du pixel $s$. II s'agit de la moyenne des dénivelés entre $s$ et les pixels voisins (en 8-connexité, noté $E$ ).

$$
\forall s, \quad \mathcal{R}(s)=\sqrt{\left.\sum_{q \in E}(M(q)-M(s))^{2}\right)},
$$

même si ces deux attributs s'avèrent très discriminants, ils ne sont pas suffisants puisque certains bas-côtés de routes et autres étendues planes ne peuvent être filtrées (Chen et al., 2014). Pour cela, on utilise un critère de pente à deux échelles que l'on appelle gradient de pente $\mathcal{G}$. Il permet d'analyser la variation de la pente sur un voisinage proche $d_{1}$ par rapport à un voisinage plus large $d_{2}$. C'est un bon indicateur d'une route en montagne (Figures 3a-c) sans avoir besoin d'avoir de franches ruptures de pente pour chaque bordure de route comme pratiqué dans la littérature de détection de réseaux ou d'intégrer des attributs de dispersion altimétrique ou de réflectance (végétation basse en bords de route etc. (David et al., 2009; Djuricic et Hollaus, 2013)). Ainsi, on a :

$$
\forall s, \quad \mathcal{G}(s)=\mathcal{M}_{d_{2}}(s)-\mathcal{M}_{d_{1}}(s),
$$

avec :

$$
\begin{aligned}
\mathcal{M}_{d_{i}}(s) & =\frac{1}{\operatorname{card} E_{d_{i}}} \sum_{q \in E_{d_{i}}} \mathcal{P}(q), i=1 \text { ou } 2, \\
E_{d_{i}} & =\left\{n \quad \mid\|s-n\|_{2} \leq d_{i}\right\} .
\end{aligned}
$$

En pratique, les $\mathcal{M}_{d_{i}}$ sont des filtres moyens sur le MNT avec des voisinages de taille différente $\left(d_{1}=2 \mathrm{~m}\right.$ et $d_{2}=12 \mathrm{~m}$, cf. Section 2.1).

\section{Masque de focalisation}

Une classification supervisée à la résolution du MNT est initiée en prenant en compte les attributs calculés à l'étape précédente et un ensemble de points d'apprentissage route et non-route, sélectionnés manuellement (cf. Partie 3). Nous choisissons la méthode des Forêts Aléatoires (Random Forests ou encore RF, (Breiman, 2001)), qui constitue aujourd'hui l'une des méthodes les plus performantes de classification pour des données de grande dimension et nécessitant un haut degré de généralisation (Criminisi et Shotton, 2013). Elles sont également peu sensibles au bruit et aux données erronées. En effet, les RF offrent l'avantage de pouvoir tolérer quelques exemples mal classés dans l'ensemble d'apprentissage sans toutefois avoir un modèle de bruit explicite, ce qui facilite la phase de sélection des points d'apprentissage.

Les RF constituent un ensemble de classifieurs supervisés (arbres de décision). L'approche consiste à générer des sous-ensembles aléatoires de données d'apprentissage pour chaque arbre (type bagging). A partir de chaque échantillon, un arbre de décision CART (Classification And Regression Trees) est construit et uniquement un sous-ensemble aléatoire de $n_{a}$ attributs est utilisé pour la division de chaque nœud. Pour une instance, chaque arbre renvoie un vote unitaire. L'étiquette finale est obtenue par vote majoritaire de tous les arbres.

Par ailleurs, le classifieur RF permet de calculer une marge d'ensemble mesurant sa fiabilité. Une marge non supervisée est proposée ici et correspond à la différence du nombre de votes de chacune des classes normalisée par le total des votes. La marge varie entre -1 et +1 . De fortes valeurs positive et négative de marge indiquent une forte confiance du classifieur respectivement pour les classes non-route et route. Une valeur de marge proche de 0 indique une forte incertitude du classifieur. 


\subsection{Construction du graphe de route}

\section{Détection des nœuds}

Les nœuds du graphe sont sélectionnés dans le masque de focalisation en suivant deux critères : on souhaite (i) les positionner dans les zones de plus forte confiance de classification RF et (ii) les répartir de manière homogène dans l'espace pour construire le graphe le plus complet possible. On utilise la technique des processus ponctuels (Descombes, 2011), issus de la géométrie stochastique, pour résoudre ce double problème de comptage d'objets et d'estimation de paramètres (leur position) (Lacoste et al., 2005; Chai et al., 2013).

Ici les objets d'intérêt sont des points 2D dont les paramètres sont les coordonnées $(x, y)$ dans le masque. La configuration $\mathbf{p}$ d'objets la plus favorable parmi toutes les configurations possibles $\mathcal{P}$ est obtenue par minimisation de l'énergie suivante $U$ :

$$
\forall \mathbf{p}, U(\mathbf{p})=\sum_{p_{i} \in \mathbf{p}} D\left(p_{i}\right)+\sum_{p_{i} \sim p_{j}} V\left(p_{i}, p_{j}\right)
$$

oé $\sim$ dénote une relation symétrique de voisinage entre objets, $D\left(p_{i}\right)$ est un terme unaire qui évalue si l'objet courant $p_{i}$ se situe sur une zone de forte marge RF, et $V\left(p_{i}, p_{j}\right)$ est un terme d'interaction binaire entre objets voisins qui permet d'éviter d'avoir deux objets trop proches spatialement. En effet, on a :

$$
\begin{gathered}
p_{i} \sim p_{j}=\left\{\left(p_{i}, p_{j}\right) \in \mathbf{p} \quad \mid\left\|p_{i}-p_{j}\right\|_{2} \leq d_{v}\right\}, \\
D\left(p_{i}\right)=1-\operatorname{marge}\left(x_{p_{i}}, y_{p_{i}}\right), \\
V\left(p_{i}, p_{j}\right)=\mathbb{1}_{\left\{\left\|p_{i}-p_{j}\right\|_{2} \geq d_{r}\right\}},
\end{gathered}
$$

oé 1 est la fonction caractéristique et $d_{v}$ et $d_{r}$ deux paramètres définissant respectivement la taille du voisinage pour l'analyse contextuelle et la distance de répulsion minimale entre deux nœuds. En pratique, on a $d_{v}=100 \mathrm{~m}$ et $d_{r}=12 \mathrm{~m}$ (cf. Partie 2.1).

Les détails relatifs à la simulation de ces processus ponctuels pour obtenir la configuration minimisant $U$ sont décrits dans (Descombes, 2011).

\section{Choix des arêtes}

Chaque nœud est ensuite relié à ses voisins immédiats en 8-connexité (ici les huit directions cardinales), si la distance avec les voisins est inférieure à 50 mètres. Cela veut dire que certains nœuds peuvent être de degré inférieur à 8 . Une distance de $50 \mathrm{~m}$ est choisie car elle permet de relier les nœuds sur un même tronçon de route sans agréger ceux sur des tronçons voisins, même en cas d'occlusion par la végétation. Nous aurions aussi pu conserver ces grandes arêtes et les filtrer à l'étape suivante mais en pratique, ce choix réduit le temps de calcul de notre méthode.

\section{Elagage des arêtes}

Le graphe ainsi établi est un ensemble exhaustif de tronçons de route potentiels. On s'intéresse à éliminer les arêtes qui ne sont pas pertinentes en se fondant sur l'hypothèse que celles qui traversent des zones avec des fortes ruptures de pente ont peu de chances de représenter une route. Pour cela, on assigne un poids à chaque arête selon deux des attributs calculés plus haut, afin d'évaluer si elle est sur une route ou pas.

Soit $\left(p_{i}, p_{j}\right)$ l'arête reliant les nœuds $p_{i}$ et $p_{j}$. On a :

$$
\begin{aligned}
& w_{\mathcal{P}}\left(p_{i}, p_{j}\right)=\frac{1}{\operatorname{card} E_{\mathcal{P}}} \sum_{s \in E_{\mathcal{P}}} \mathcal{P}(s), \\
& w_{\mathcal{G}}\left(p_{i}, p_{j}\right)=\frac{1}{\operatorname{card} E_{\mathcal{G}}} \sum_{s \in E_{\mathcal{G}}} \mathcal{G}(s),
\end{aligned}
$$

oé $E_{\mathcal{P}}$ et $E_{\mathcal{G}}$ sont respectivement l'ensemble des pixels de $\mathcal{P}$ et $\mathcal{G}$ intersectés par l'arête $\left(p_{i}, p_{j}\right) .\left(p_{i}, p_{j}\right)$ est éliminée si :

$$
w_{\mathcal{P}}\left(p_{i}, p_{j}\right) \geq t_{\mathcal{P}} \vee w_{\mathcal{G}}\left(p_{i}, p_{j}\right) \leq t_{\mathcal{G}}
$$

La décision de conserver ou non les arêtes est prise automatiquement en fonction de l'ensemble d'apprentissage saisi manuellement pour la classification supervisée RF. II permet de saisir l'hétérogénéité géométrique des routes et nous donne une bonne indication des valeurs des pixels (pente et gradient de pente) pour lesquelles on peut admettre que des tronçons de route existent. En pratique, les deux seuils valent $t_{\mathcal{P}}=30$ et $t_{\mathcal{G}}=50$ environ.

\subsection{Caractérisation 3D}

Cette dernière étape consiste à estimer la largeur et l'inclinaison des routes en utilisant la carte de pente $\mathcal{P}$, tout en éliminant les candidats irréalistes. En effet, un certain nombre d'éléments du terrain se comporte géométriquement comme des routes (zones homogènes planes) mais ne doit pas être conservé : zones naturelles, terrassements, tout comme le terrain sous couvert forestier, qui par manque de points lidar, peut avoir été interpolé par un grossier plan horizontal. Pour cela, la caractérisation et le filtrage 3D des routes se décomposent en deux étapes.

\section{Analyse de profils}

Tout d'abord, chaque arête est analysée afin d'en déduire la véritable zone de route qui la supporte. Le parcours de toutes les arêtes permet de générer une nouvelle carte binaire $\mathcal{B}$ route/non-route, à la résolution de $1 \mathrm{~m}$, sur laquelle sera fondée l'analyse orientée-objet. Pour cela, chaque arête est discrétisée à un pas de $1 \mathrm{~m}$. Ensuite, pour chaque nouveau sommet, on évalue le profil de pente, orthogonalement à la direction de l'arête. On étend ce profil jusqu'à une distance $d_{2}=12 \mathrm{~m}$ tant que l'inclinaison entre deux pixels consécutifs est inférieure à $t_{\mathcal{P}}^{\perp}=10$ (appris sur l'ensemble utilisé pour la classification). Enfin, tous les pixels de $\mathcal{B}$ intersectant ces profils générés sont assignés à la valeur 1 . 


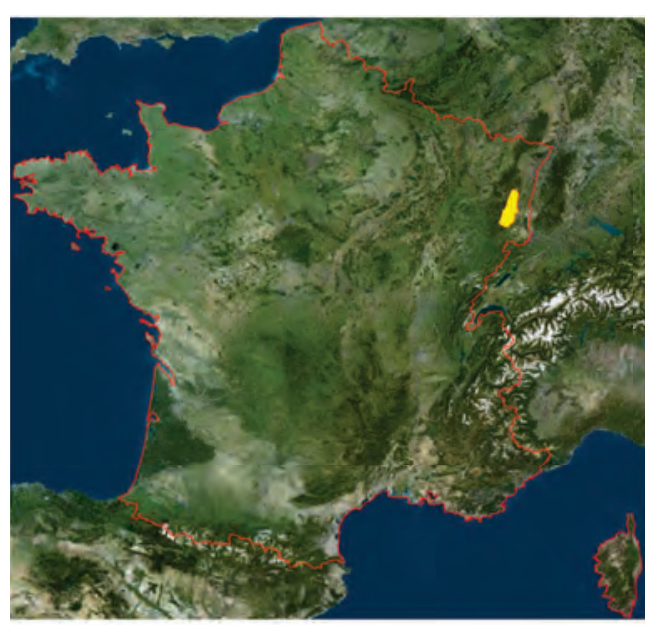

(a)

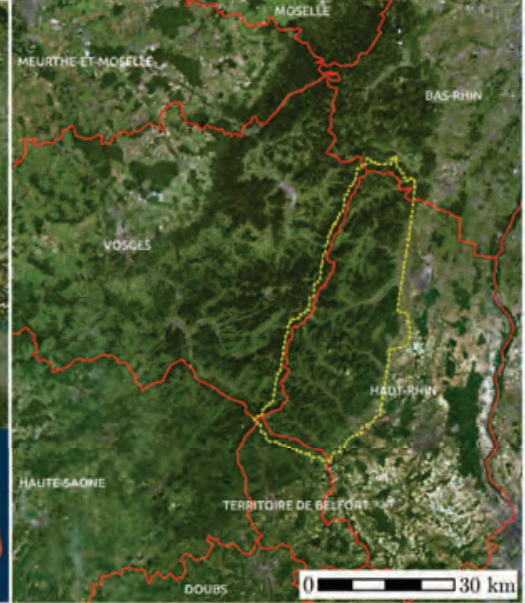

(b)

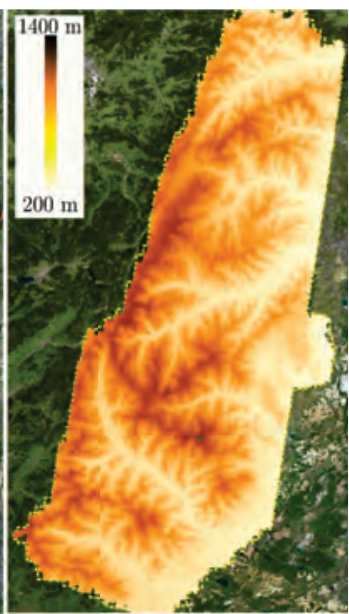

(c)

FIGURE 4 : Aperçu de la zone d'intérêt sur les Vosges alsaciennes (en jaune). (a) Vue France entière. (b) Emprise des données lidar. (c) Modèle Numérique de Terrain lidar (5 $\mathrm{m}$ de résolution).

\section{Analyse orientée objet et estimation géométrique}

Tout d'abord, des objets tronçons de route sont extraits de $\mathcal{B}$ par une segmentation en composantes connexes en 8-connexité (cf. Figures 3e-f), afin de pouvoir réaliser une analyse géométrique orientée objet (OBIA).

On utilise pour cela un attribut de texture : Structural Feature Set (SFS, (Huang et al., 2007)) qui permettent d'extraire des informations statistiques dans l'histogramme des extensions des lignes de direction d'une image. Une extension d'une ligne dans une direction donnée est définie comme la mesure d'un ensemble de pixels passant par le pixel central et dont la différence des niveaux de gris est inférieure à un seuil donné. Différents attributs peuvent être extraits de cet histogramme : nous utiliserons la longueur et la largeur. La méthode SFS a été adaptée ici afin de traiter l'image binaire $\mathcal{B}$, ce qui permet de s'affranchir du seuil. Pour réduire les temps de calcul et être cohérent avec les étapes précédentes (cf. Sections 2.2 et 2.3), nous avons choisi de limiter l'analyse aux 8 directions principales et à une longueur maximale d'analyse de $50 \mathrm{~m}$ (Section 2.3).

Ensuite, pour éliminer les erreurs résiduelles provenant principalement de petites zones planes sous couvert forestier, les objets dont le ratio longueur/largeur est inférieur à 10 sont éliminés (Figures $3 e$ and f).

La pente de chacun des tronçons finaux est enfin estimée directement à partir du Modèle Numérique de Terrain.

\section{Données}

La performance de la méthode a été évaluée sur une zone d'étude de près de $1425 \mathrm{~km}^{2}$. Elle couvre une partie des Vosges alsaciennes, à cheval sur quatre départements français, mais principalement le Haut-Rhin (68). L'altitude du terrain varie entre $200 \mathrm{~m}$ et $1365 \mathrm{~m}$.
Le couvert forestier est principalement composé d'épiceas, de pins sylvestres, d'érables sycomores, d'hêtres et de chênes cécilles. La campagne d'acquisition lidar a été menée par l'Institut National de l'Information Géographique et Forestiére (IGN) en avril 2011 avec un capteur Optech 3100EA. L'angle de balayage était de $\pm 17^{\circ}$ pour une tâche au sol de $0,8 \mathrm{~m}$ environ. La densité de points $3 \mathrm{D}$ au sol varie entre 2 et $4 \mathrm{pt} / \mathrm{m}^{2}$, ce qui coïncide parfaitement avec les spécifications opérationnelles d'acquisitions en zones forestières sur de très grandes surfaces, telles que pratiquées dans de nombreux pays (Maltamo et al., 2014), y compris pour l'inventaire forestier (McRoberts et Tomppo, 2007).

Prés de 5,6 milliards de points 3D sont ainsi disponibles et la zone d'étude a été découpée en dalles régulières de $1 \mathrm{~km} \times 1 \mathrm{~km}$ pour faciliter les traitements. Chacune des dalles a subi une étape de filtrage des points du sursol puis d'interpolation afin de générer des Modèles Numériques de Terrain de résolution $1 \mathrm{~m}$. Ces traitements sont des routines de l'IGN pour l'amélioration de la référence altimétrique nationale. Ils sont issus du logiciel TerraSolid (Soininen, 2014) et ne sont pas remis en cause ni analysés dans cette étude.

Les données d'apprentissage pour la classification (Partie 2.2) représentent une vingtaine de polygones sélectionnés manuellement sur quelques dalles ( $\sim 16250$ pixels, soit $9,4.10^{-6} \%$ de la surface totale). Ce jeu est suffisant pour obtenir un bon masque de focalisation sur l'intégralité de la zone d'étude et l'estimation de la plupart des paramètres.

Deux bases de données (BD) topographiques 2D de routes sont disponibles sur notre zone d'étude : la première provient de l'IGN et la seconde de l'Office National des Forêts (ONF). Elles ont été obtenues de manière indépendante par une combinaison de photo-interprétation et de relevés terrain. Elles sont toutes les deux très exhaustives, y compris sous couvert forestier dense, mais souffrent d'un manque de précision géométrique quant 
à la localisation des objets la composant. Cette imprécision spatiale empêche une comparaison directe entre nos résultats et ces données de référence, c'est pourquoi une vérité terrain complémentaire, représentant $320 \mathrm{~km}$ de routes, a été saisie manuellement sur près de $100 \mathrm{~km}^{2}$ par analyse visuelle du Modèle Numérique de Terrain lidar et des orthoimages disponibles. Ainsi, les BD IGN et ONF serviront uniquement à une qualification visuelle des résultats (partie 4.1) alors que la saisie manuelle sera employée pour une qualification chiffrée des performances de notre méthode (partie 4.2). Ces deux BD possèdent également des informations sémantiques sur les types de route ainsi que de largeur. Toutefois, on se trouve confronté à deux problémes : d'une part, la nomenclature proposée n'est pas compatible avec celle établie pour la mobilisation du bois et, d'autre part, un grand nombre de routes ont leur attribut de largeur égal à 0 (cf. Figure 1).

\section{Résultats et discussions}

\subsection{Analyse qualitative}

Les principaux avantages de la méthode, outre sa rapidité (Partie 4.4) et ses aspects logiciels (briques open-source facilement modifiables), sont sa capacité (i) à estimer la largeur des routes (Figure 3), (ii) à détecter des routes non présentes dans les BD et (iii) à localiser les routes de manière plus précise que dans les BD métiers (Figures 5 et 6 ). Le fait de fonder intégralement notre analyse sur la morphologie des MNT lidar permet de s'affranchir de ces problèmes.

Les routes non présentes dans les deux BD analysées varient d'une base à une autre et correspondent autant à des objets sous couvert végétal dense qu'à des pistes sur terrains nus, que l'on ne peut pas détecter par photo-interprétation dans la plupart des cas (Figure 6a). II ne s'agit jamais de petits tronçons de route (ou de fin de routes) mais de grandes portions de plus de $100 \mathrm{~m}$, ce qui rend notre méthode attractive malgré la faible proportion du réseau non existant sur notre zone d'étude (5-10\%). II faut noter toutefois que les Vosges constituent un territoire dont la desserte est décrite de manière bien plus exhaustive dans ces BD que d'autres zones en France.

De plus, la méthode permet de repositionner les routes existantes de l'ordre de 5-10 m (décalage dé à la méthode de saisie initiale ou au processus de généralisation sous-jacent (Fujimura et al., 2008)), qui varie selon les tronçons et qui existe pour près de $40 \%$ des routes analysées (Figure 6). La pertinence du lidar pour une bonne localisation a déjà été démontrée dans (White et al., 2010) et (Azizi et al., 2014). En outre, cet aspect permet d'envisager notre méthode, dans le cas d'un réseau routier filaire déjé exhaustif, comme un outil automatique de repositionnement de graphes routiers existants et de caractérisation géométrique (en sautant l'étape 2, Section 2.1 et figure 2). De surcroît, des détails fins sur les tronçons (virages) non présents dans les BD sont également restitués (Figure 6). Ils sont avant tout importants pour estimer de manière précise les rayons de courbure des tronçons de route, nécessaire pour une bonne sémantisation de la desserte en fonction des véhicules qui l'empruntent.

Les faiblesses de notre méthode sont de deux types. Tout d'abord, elle est fondée sur l'analyse du gradient de la pente et donc ne permet pas d'extraire des routes sur certains milieux. Seul un critère d'élongation permet de filtrer les terrains "plats non routes", ce qui n'est pas suffisant pour séparer les tronçons des routes des zones urbaines, des bas-cétés ou d'aires de retournement et de débardage (cf. gauche de la figure 3). On observe donc à ces endroits des sur-détections tout comme dans certains thalwegs, qui peuvent avoir une morphologie identique aux routes. En pratique, si nous avons les BD adéquates à disposition (hydrologie et bâtiments), un masque peut être créé et permet de réduire drastiquement ces erreurs inhérentes à la méthodologie (Figure 6c). D'autres sur-détections provenant d'activités humaine intensive (labourage, irrigation, terrasses), y compris dans des zones de forêts nouvellement plantées, peuvent elles être plus difficilement corrigées sans information contextuelle et forts a priori géométriques.

Ensuite, des sous-détections sont observées quand la géométrie du terrain ne suit pas le modèle proposé. On le note pour certains tronçons de route où le MNT ne présente pas de pente spécifique. Cela est dû d'une part à une reconstruction du terrain localement limitée à un trop faible nombre de points lidar au sol (e.g., sous couvert forestier dense) et à l'élagage du graphe. En effet, si le nœud d'une arête se situe légèrement en dehors de la route, cette dernière aura des valeurs de $w_{\mathcal{P}}$ ou de $w_{\mathcal{G}}$ élevées (Equation 11) et ne sera pas conservée (Figure 5). Le processus de classification est assez simple. II en résulte une zone de probabilité route élevée de part et d'autre du tronçon et dans le processus de détection des nœuds, aucune contrainte géométriquement n'oblige un nœud à être aligné à deux voisins : cela permet d'avoir des nœuds dans les virages mais également légérement sur le bas-cété des routes. Nous aurions pu fixer une valeur de $d_{r}$ plus petite mais cela aurait généré un nombre de nœuds bien plus grand et un processus plus coûteux en temps. De méme, en cas d'occlusion et en l'absence de méthodologie adaptée, les arêtes générées sont exclues de l'analyse 3D car elles ne remplissent aucun critère.

De plus, les jonctions ne sont pas intégrées au processus ponctuel ainsi que dans l'analyse OBIA, là même où le modèle de route proposé et les critéres géométriques afférents ne sont plus valides. Ainsi, sans analyse de plus haut niveau, ces zones sont souvent mal détectées, un tronçon de route étant privilégié par rapport aux autres (Figure 6c). 

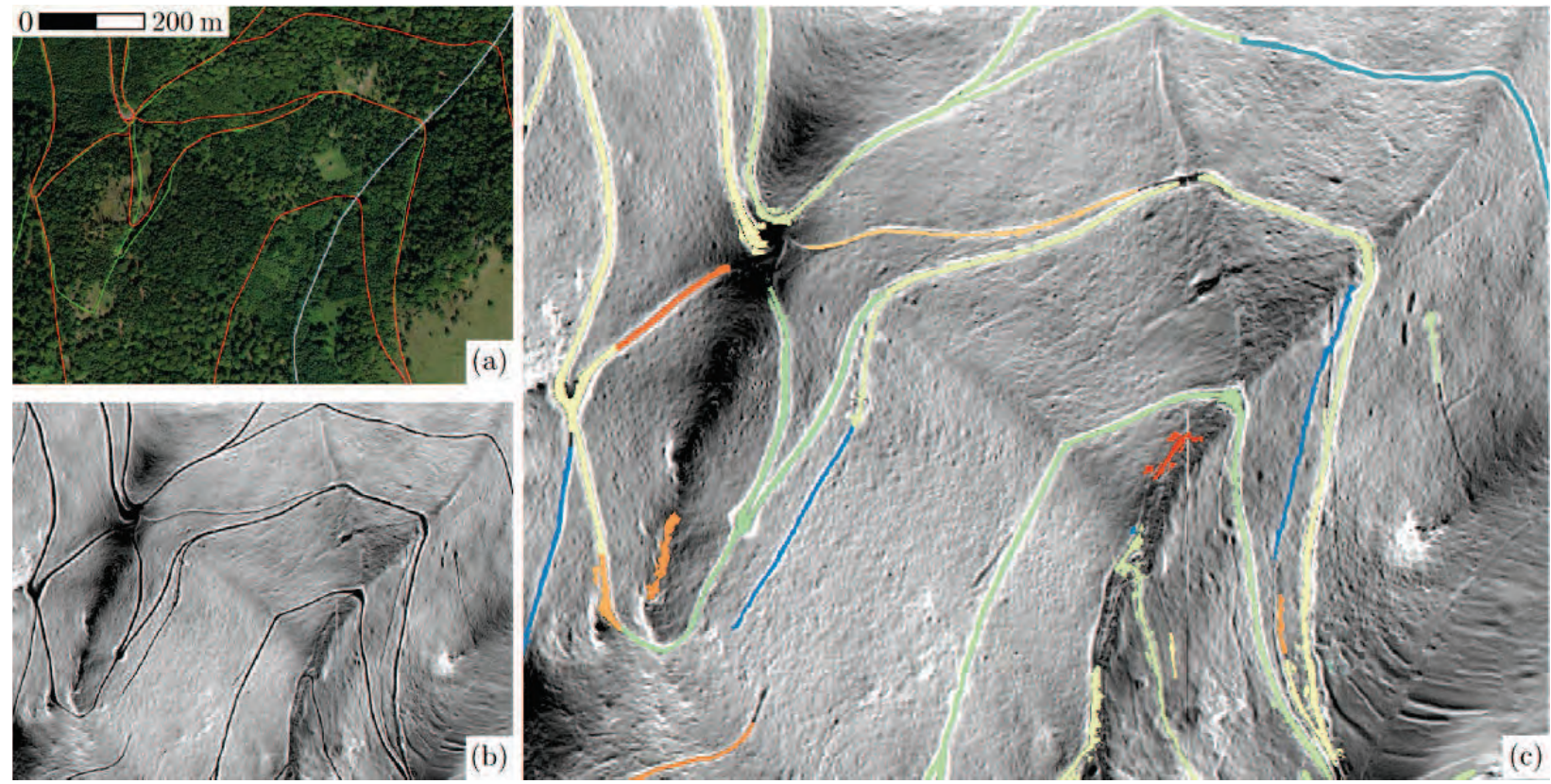

FIGURE 6 : Avantage et inconvénients de la méthode proposée. (a) Orthoimage de la zone avec deux bases de données de dessertes existantes (rouge et vert) et le réseau hydrographique (bleu) superposés. (b) La carte de pente $\mathcal{P}$ : on peut y noter la trés bonne restitution de la desserte, les manques des BD existantes, ainsi que les décalages planimétriques existants avec la réalité. (c) Notre résultat (code couleur identique à la figure 3). On y voit la bonne qualité de détection (localisation et exhaustivité), les problèmes aux jonctions des routes et une détection partielle du réseau hydrographique.

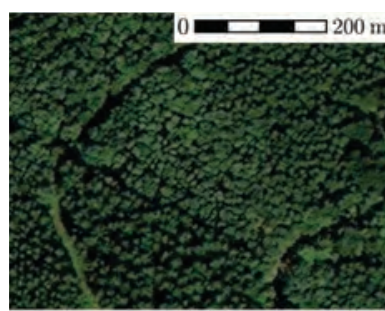

(a)

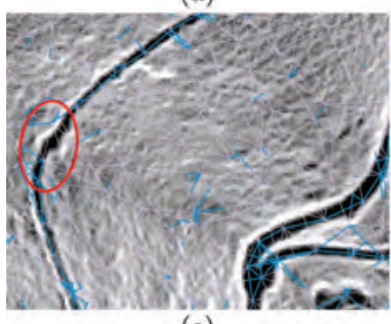

(c)

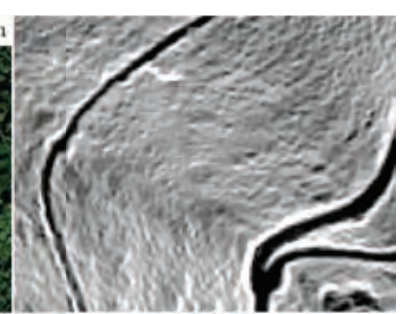

(b)

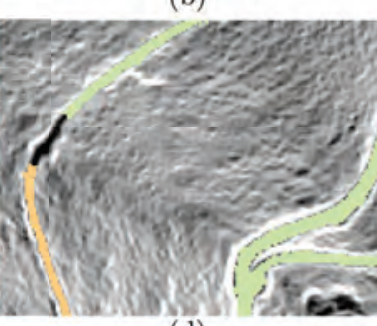

(d)
FIGURE 5 : Illustration du manque d'exhaustivité locale dans la détection. (a) Orthoimage. (b) Carte de pente $\mathcal{P}$. (c) Graphe avant et après élagage (respectivement en rose et bleu). (d) Résultat final (code couleur identique à la figure 3).

\subsection{Analyse quantitative}

L'évaluation de la détection des routes est réalisée par la méthode proposée par (Heipke et al., 1997), en comparant le réseau détecté à un réseau de référence vectorisé. Les indicateurs de performance sont fondés sur des longueurs de routes. Cette méthode peut être appliquée aux réseaux connectés ou déconnectés comme dans notre cas. Le processus d'appariement est fondé sur la recherche, dans une zone tampon autour de la route de référence, des parties de routes détectées correspondantes.

D'abord, un tampon de largeur constante prédéfinie est construit autour des données de route de référence. Les routes détectées appariées correspondent aux vrais positifs avec la longueur TP1 tandis que celles non appariées sont appelées des faux positifs avec une longueur $F P$. Le processus est ensuite appliqué, dans l'autre direction, en prenant les routes détectées comme référence. On obtient alors TP2, la longueur des routes de référence qui correspondent au routes détectées. Les données de référence non appariées sont appelées faux négatifs et ont une longueur notée $F N$. La largeur de tampon a été fixée à $5 \mathrm{~m}$ afin de prendre en compte les différentes précisions planimétriques des routes détectées et celles de référence (cf. Section 4.1).

Quatre paramètres habituels sont donc obtenus; la métrique vrai positif TP qui est la moyenne de TP1 et TP2, les faux positifs FP (erreurs de commission), les vrais négatifs $T N$ (erreur d'omission) qui est la longueur des données de référence qui ne sont pas inclus dans le réseau détecté. Ces métriques sont normalisées par 


\begin{tabular}{|c|c|c|c|c|}
\hline & TP & FP & TN & ERR \\
\hline $\mathrm{L}$ & 0,9 & 0,17 & 0,1 & 0,27 \\
\hline $\mathrm{L}>1 \mathrm{~m}$ & 0,9 & 0,17 & 0,1 & 0,27 \\
\hline $\mathrm{L}>2 \mathrm{~m}$ & 0,88 & 0,11 & 0,12 & 0,23 \\
\hline $\mathrm{L}>3 \mathrm{~m}$ & 0,82 & 0,10 & 0,18 & 0,28 \\
\hline
\end{tabular}

TABLEAU 1 : Influence de la longueur minimale des routes détectées sur leur évaluation.

\begin{tabular}{|c|c|c|c|c|}
\hline Taille zone tampon & TP & FP & TN & ERR \\
\hline $1 \mathrm{~m}$ & 0,58 & 0,24 & 0,42 & 0,66 \\
\hline $2 \mathrm{~m}$ & 0,78 & 0,13 & 0,22 & 0,35 \\
\hline $3 \mathrm{~m}$ & 0,84 & 0,12 & 0,16 & 0,28 \\
\hline $4 \mathrm{~m}$ & 0,86 & 0,11 & 0,14 & 0,25 \\
\hline $5 \mathrm{~m}$ & 0,88 & 0,11 & 0,12 & 0,23 \\
\hline
\end{tabular}

TABLEAU 2 : Influence de la taille de la zone tampon sur l'évaluation des routes.

la longueur du réseau routier de référence et varient donc entre 0 et 1 . Enfin la somme des erreurs est notée $E R R=F P+T N$.

$320 \mathrm{~km}$ de routes ont été saisis manuellement. Sur la figure $7(1)$ et dans le tableau 1 , on voit que les taux de vrais positifs sont très bon et varient de $90 \%$ à $82 \%$ sans élagage et avec un élagage important de tous les segments inférieurs à $3 \mathrm{~m}$. On remarque que dans ce dernier cas, le taux TN (vrais négatifs) augmente et des parties de routes sont manquées. Au contraire, on remarque qu'avec un faible élagage, le taux $f p$ est supérieur induisant beaucoup de fausses détections essentiellement dues aux segments de petites tailles. La figure 7(2) et le tableau 2 montrent l'évolution des métriques d'évaluations en fonction de la taille de la zone tampon. Plus la taille de la zone tampon augmente et plus les performances de détection sont améliorées. Ceci est essentiellement dû aux problèmes de précision planimétrique entre les deux réseaux routiers. Finalement, en observant ces deux figures, le meilleur compromis est obtenu avec un rayon de zone tampon de $5 \mathrm{~m}$ et un élagage des segments de routes qui sont inférieurs ou égaux à $2 \mathrm{~m}$ de longueur (Tableau 3).

On retrouve sur la figure 8 les principales constatations dressées dans la partie précédente : les faux positifs sont des petites zones de terrain nu, souvent proche des routes, et les faux négatifs correspondent principalement à des occlusions et à des jonctions entre plusieurs

\begin{tabular}{|c|c|c|}
\hline & \multicolumn{2}{|c|}{ Classification } \\
\hline Vérité Terrain & Route & Non-Route \\
\hline Route & $\mathrm{TP}=0,88$ & $\mathrm{FP}=0,12$ \\
\hline Non-Route & $\mathrm{FN}=0,12$ & $\mathrm{TN}=0,88$ \\
\hline
\end{tabular}

TABLEAU 3 : Matrice de confusion du meilleur compromis obtenu pour une zone de tampon de $5 \mathrm{~m}$ et un élagage des segments dont la longueur est inférieure ou égale à $2 \mathrm{~m}$.

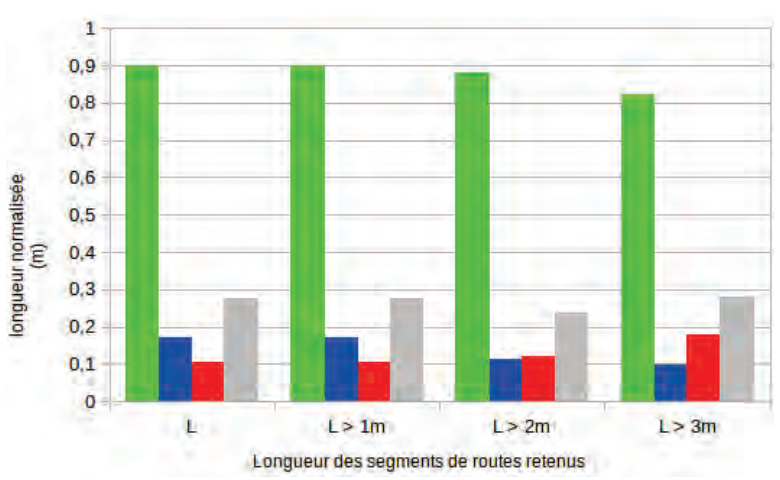

(1) Influence de la longueur minimale de la route détectée.

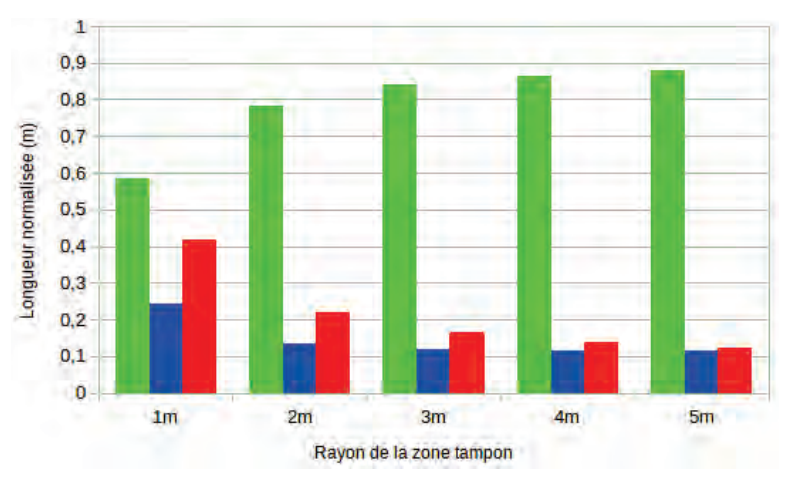

(2) Influence de la taille de la zone tampon.

FIGURE 7 : Influence des paramétrages d'évaluation. $\square:$ TP $-\square:$ FP - $\mathbf{a}:$ TN - $\mathbf{a}:$ ERR.

routes.

\subsection{Sensibilité des paramètres mis en jeu}

Le jeu de paramètres utilisé pour les traitements est le même pour toutes les dalles de la zone d'intérêt. Ceuxci sont de trois types dans notre étude :

- Ceux appris à partir de l'ensemble d'entraînement saisi manuellement (cf. Partie 3), en particulier pour l'élagage du graphe.

- Ceux du modèle de route.

- Ceux fixés pour contrôler les temps de calcul et garantir une capacité de traitements sur de grandes surfaces.

Concernant les premiers, nous n'avons pas noté une meilleure qualité de détection de la desserte à proximité de la zone d'apprentissage et la décimation du graphe se déroule de manière identique partout. Cela nous permet de dire que ce jeu permet de fixer les paramètres $t_{\mathcal{P}}, t_{\mathcal{G}}$ et $t_{\mathcal{P}}^{\perp}$ pour toute la zone sans réglage supplémentaire et sans gain/perte de qualité.

Ensuite, les paramètres $d_{1}$ et $d_{2}$ ont été établis via la définition du modèle de route en entrée. Ce sont les bornes minimales et maximales d'analyse. Elles ont été sélectionnés afin de capturer les variations du relief entre deux échelles tout en s'assurant, pour les pixels route, que la première corresponde à la route et la seconde n'y corresponde pas. Une plus petite valeur de $d_{1}$ filtrerait ainsi les routes peu larges car la carte de pente multi-échelles fonctionnerait comme un filtre moyen lissant la carte de 

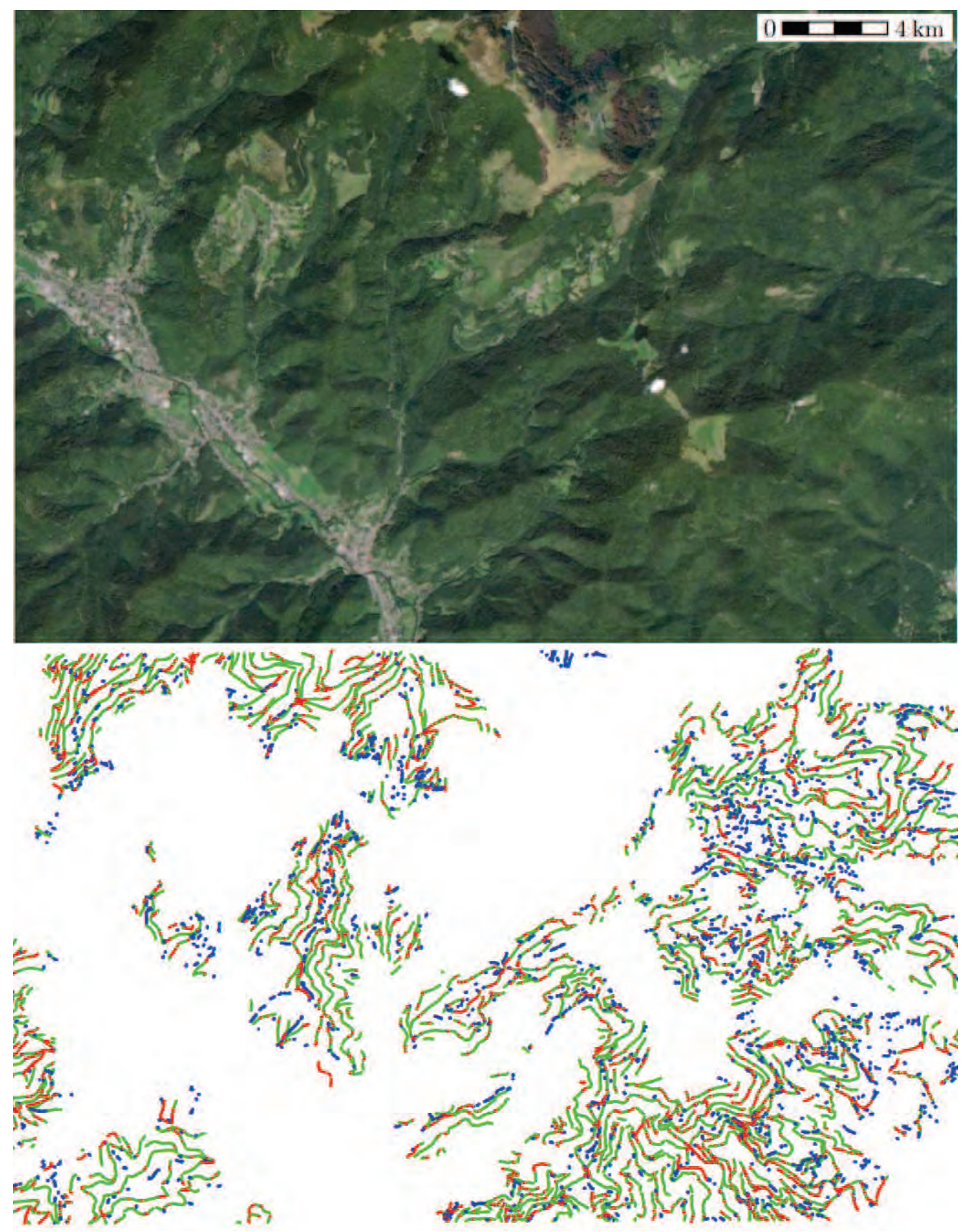

FIGURE 8 : Résultats d'évaluation de la détection de routes sur la zone du Grand Ballon : rayon buffer $=5 \mathrm{~m}$, longueur minimale $=3 \mathrm{~m}$. Haut : orthoimage. Bas : qualification. Légende identique à la figure 7. 


\begin{tabular}{c|l|c} 
Etape & Traitement & Temps (s) \\
\hline \hline \multirow{4}{*}{1} & Extraction d'attributs sur MNT & $2 "$ \\
& Calcul de la différence de pentes & $6 "$ \\
& Classification supervisée & $16 "$ \\
\hline \multirow{2}{*}{2} & Processus Ponctuel & $22^{\prime \prime}$ \\
& Construction/élagage du graphe & $19 "$ \\
\hline \multirow{3}{*}{3} & Analyse de profils & $1 "$ \\
& OBIA & $36 "$ \\
& Linéarisation & $11^{\prime \prime}$ \\
& Sauvegarde de tous les attributs & $2 "$ \\
\hline \hline \multicolumn{2}{|c|}{ Total } & $1 ' 55^{\prime \prime}$
\end{tabular}

TABLEAU 4 : Temps de calcul moyen par $\mathrm{km}^{2}$ par étape de la méthode.

pente $\mathcal{P}$. De plus, cela réhausserait les zones planes non-routes. Une valeur trop faible de $d_{2}$ (e.g., $d_{2} \in[4-$ $10 \mathrm{~m}])$ ne permet pas de se focaliser sur les bons objets alors que $d_{2}=12 \mathrm{~m}$ réhausse efficacement le contraste de la carte de pente en éliminant les zones plates non-route, les artefacts du MNT et en mettant en avant les routes de différentes largeurs.

Enfin, les paramètres restants $\left(d_{r}, d_{v}\right.$ pour l'initialisation du graphe, ceux qui en découlent dans l'analyse orientée-objet et ceux du SFS) ne sont là non pas critiques puisqu'ils servent avant tout à régler les temps de calculs. Un intervalle assez large pour chacun d'entre eux est en effet valable et conduirait aux mêmes résultats de détection. On peut ainsi par exemple créer initialement plus de nœuds en réduisant $d_{r}$ ou en augmentant fortement $d_{v}$ mais ceux-ci seront éliminés lors de l'étape suivante. Cela ne fait que conduire à des temps de calcul plus importants sans augmenter la qualité de détection puisque les principaux causes identifiées de l'incomplétude du graphe (jonctions de routes et occlusions trop importantes) ne peuvent être corrigées par la création d'un réseau initial plus dense.

Finalement, l'intégralité de l'analyse se fait à la résolution des MNT en entrée. Cette valeur nous convient car puisque cela nous permet de conserver certaines hautes fréquences du relief nécessaires à la détection des routes, le nombre de pixels initial pour la classification par Forêts Aléatoires n'empêche pas un étiquetage rapide et nous obtenons des estimations de la largeur des routes en accord avec les spécifications fournies par les professionnels de la mobilisation du bois en zones forestières.

\subsection{Temps de calcul}

Les temps de calcul de la méthode ont été examinés sur plus de 70 dalles d'un kilomètre carré, centrées sur la zone du Grand Ballon, point culminant des Vosges, afin de s'assurer une grande diversité de pentes, de densités de couvert forestier et de dessertes. Les chiffres par étape sont détaillés dans la Table 4 pour un unique processeur de $3,3 \mathrm{GHz}$. Ils correspondent aux moyennes sur toutes les dalles analysées. On obtient un temps de trai- tement de moins de 2 minutes par $\mathrm{km}^{2}$ ce qui est très satisfaisant et provient du fait (i) que l'on a évité d'impliquer des processus demandant beaucoup de ressources et/ou (ii) que l'on a fondé la plupart de nos implémentations sur des librairies existantes et connues pour leur efficacité : Sherwood, LibRJMCMC, OTB et CGAL.

Les trois étapes les plus coûteuses sont la classification pixellaire initiale, la détection des nœuds initiaux du graphe et l'analyse orientée objet. Le temps de classification peut être réduit en adoptant une implémentation parallélisée des Foréts Aléatoires. La détection des nœuds initiaux par Processus Ponctuels est déjà optimisée et correspond à 17 millions d'itérations du processus stochastique. Cette technique est connue pour converger lentement et ainsi être relativement lentes, et donc non compatibles avec des problématiques de passage à l'échelle. Toutefois, nous utilisons l'implémentation disponible dans la LibRJMCMC (Brédif et Tournaire, 2012) (couplée à une formulation énergétique simple requérant peu d'opérations mathématiques) qui permet d'effectuer ces itérations nécessaires en moins de 30 secondes $/ \mathrm{km}^{2}$.

II n'est pas nécessaire de l'améliorer tant que la formulation énergétique sous-jacente reste simple. Enfin, la plupart des temps de calcul de l'analyse orientée objet provient du calcul de l'attribut de texture SFS (Structural Feature Set) qui est l'un des attributs les plus pertinents en télédétection pour la détection des routes dans des images très haute résolution spatiale.

\section{Conclusions}

La méthode proposée, la première à cette échelle, a permis de détecter de manière totalement automatique la desserte forestière en milieu montagneux avec une exhaustivité élevée. L'approche a été développée pour être rapide et a utilisé pour cela des implémentations efficaces d'outils de classification et de reconnaissance des formes. Elle se compose de deux parties disjointes de détection et de caractérisation 3D. La deuxiéme étape peut également être considérée pour le recalage de BD de routes existantes exhaustives mais dont la précision géométrique est insuffisante.

Les uniques paramètres en jeu sont les bornes minimales et maximales acceptables de route. L'apprentissage initial sert à régler tous les autres ainsi que le compromis entre fort taux de bonnes détections et faible taux de fausses détections. L'approche n'utilise par ailleurs que des outils open source : elle peut donc être reproduite et améliorée brique à brique. Cela est d'autant plus vrai que notre raisonnement n'est fondé que sur la connaissance du modèle Numérique de Terrain lidar et d'un modèle géométrique simple de route. Ceci constitue également la principale faiblesse actuelle de l'approche : se limiter à une analyse 2D sur des attributs issus du MNT empêche d'extraire des informations du nuage de points 3D brut qui auraient pu permettre de se focaliser plus efficacement sur les zones routes (en éliminant 
certains thalwegs) ou d'éliminer certaines arêtes irréalistes dans le graphe (présence de végétation basse par exemple).

Les perspectives sont de deux types. Tout d'abord, elles sont méthodologiques. La détection pourrait être améliorée en intégrant à notre méthode ascendante des approches descendantes plus complexes tant pour la classification (Wegner et al., 2013; Montoya et al., 2014) que pour la construction du graphe (Chai et al., 2013). Elle pourrait l'être également en introduisant des connaissances métiers actuelles (couvert forestier, revêtement...). Ensuite, d'un point de vue opérationnel, il faudrait être capable de qualifier la détection effectuée pour signaler à un opérateur les tronçons potentiellement erronés ainsi que ceux non détectés mais qui devraient être analysés a posteriori. Une telle intégration dans une boucle de traitement permettra de réduire l'influence de l'apprentissage initial et limiter drastiquement le taux de fausses détections.

\section{Remerciements}

Cette étude a été financée par l'Agence Nationale de la Recherche (ANR) à travers le projet FORESEE (ANR-2010-BIOE-008) mais également par la Fondation Portugaise pour la Science et la Technologie (PEst-OE/EEI/UI308/2014). Les auteurs remercient Jean-Stéphane Bailly (UMR LISAH) pour avoir fourni le code d'évaluation de détection des routes et Mathieu Brédif (IGN) pour son aide sur la LibRJMCMC.

\section{Références}

Amo, M., Martinez, F., Torre, M., 2006. Road extraction from aerial images using a region competition algorithm. IEEE Transactions on Image Processing 15 (5), 1192-1201.

Azizi, Z., Najafi, A., Sadeghian, S., 2014. Forest road detection using lidar data. Journal of Forestry Research 25 (4), 975980.

Barzohar, M., Cooper, D., 1996. Automatic finding of main roads in aerial images by using geometric-stochastic models and estimation. IEEE Transactions on Pattern Analysis and Machine Intelligence 18 (7), 707-721.

Bellier, J., Monnet, J.-M., Dupire, S., Cordonnier, T., 2014. Evaluation of the effect of accessibility on forest stand structure with airborne laser scanning data and GIS-based models. Dans : ForestSAT. 4-7 November 2014, Riva del Garda, Italy.

Borga, M., Tonelli, F., dalla Fontana, G., Cazorzi, F., 2005. Evaluating the influence of forest roads on shallow landsliding. Ecological Modelling 187 (1), 85 - 98.

Brédif, M., Tournaire, O., 2012. LibRJMCMC : An open-source generic $\mathrm{C}_{+}+$library for stochastic optimization. International Archives of the Photogrammetry, Remote Sensing and Spatial Information Sciences XXXIX-B3, 259-264.

Breiman, L., 2001. Random forests. Machine Learning 45 (1), 5-32.

Chai, D., Forstner, W., Lafarge, F., 2013. Recovering linenetworks in images by junction-point processes. Dans : IEEE conference on Computer Vision and Pattern Recognition. IEEE, 23-28 June 2013, Portland, OR, USA.
Chen, W., Li, X., Wang, Y., Chen, G., Liu, S., 2014. Forested landslide detection using LiDAR data and the random forest algorithm : A case study of the Three Gorges, China. Remote Sensing of Environment 152, $291-301$.

Clode, S., Rottensteiner, F., Kootsookos, P., 2005. Improving city model determination by using road detection from lidar data. International Archives of the Photogrammetry, Remote Sensing and Spatial Information Sciences XXXVI(3/W24), 159164.

Clode, S., Rottensteiner, F., Kootsookos, P., Zelniker, E., 2007. Detection and vectorization of roads from lidar data. Photogrammetric Engineering \& Remote Sensing 73 (5), 571-535.

Coffin, A., 2007. From roadkill to road ecology : A review of the ecological effects of roads. Journal of Transport Geography 15 (5), 396-406.

Criminisi, A., Shotton, J. (Eds.), 2013. Decision Forests for Computer Vision and Medical Image Analysis. Springer.

David, N., Mallet, C., Pons, T., Chauve, A., Bretar, F., 2009. Pathway detection and geometrical description from ALS data in forested mountaneous areas. International Archives of Photogrammetry, Remote Sensing and Spatial Information Sciences 38 (Part 3/W8), 242-247.

Descombes, X. (Ed.), 2011. Stochastic Geometry for Image Analysis. Wiley.

Djuricic, A., Hollaus, M., 2013. Extraction of forest roads from full-waveform airborne laser scanning data. Dans : EGU. 712 April 2013, Vienna, Austria.

Eysn, L., Hollaus, M., Berger, F., 2014. NEWFOR - Enhancing the wood supply chain within the alpine space. Dans : ForestSAT. 4-7 November 2014, Riva del Garda, Italy.

Fathi, A., Krumm, J., 2010. Detecting road intersections from gps traces. Dans : Fabrikant, S. I., Reichenbacher, T., van Kreveld, M., Schlieder, C. (Eds.), Geographic Information Science. Vol. 6292 de Lecture Notes in Computer Science. pp. 56-69.

Fujimura, H., Ziems, M., Heipke, C., 2008. De-generalization of japanese road data using satellite imagery. Photogrammetrie - Fernerkundung - Geoinformation 5, 363-373.

Grote, A., Heipke, C., Rottensteiner, F., 2012. Road network extraction in suburban areas. The Photogrammetric Record 27 (137), 8-28.

Gucinski, H., Furniss, M., Ziemer, R., Brookes, M., 2001. Forest roads : A synthesis of scientific information. Rapport technique PNW-GTR-509, USDA Forest Service, Pacific Northwest Research Station : Corvallis, OR, USA.

Hatger, C., 2005. On the Use of Airborne Laser Scanning Data to Verify and Enrich Road Network Features. International Archives of Photogrammetry, Remote Sensing and Spatial Information Sciences 36 (Part 3/W19), 138-143.

Heipke, C., Mayer, H., Wiedemann, C., Jamet, O., 1997. Evaluation of automatic road extraction. International Archives of Photogrammetry and Remote Sensing XXXII (Part 3-4W2), 47-56.

Hinz, S., Baumgartner, A., 2003. Automatic extraction of urban road networks from multi-view aerial imagery. ISPRS Journal of Photogrammetry and Remote Sensing 58 (1-2), $83-98$.

Hu, J., Razdan, A., Femiani, J., Ming, C., Wonka, P., 2007. Road network extraction and intersection detection from aerial images by tracking road footprints. IEEE Transactions on Geoscience and Remote Sensing 45 (12), 4144-4157.

Hu, X., Li, Y., Shan, J., Zhang, J., Zhang, Y., 2014. Road centerline extraction in complex urban scenes from lidar data based on multiple features. IEEE Transactions on Geoscience and Remote Sensing 52 (11), 7448-7456.

Huang, X., Zhang, L., Li, P., 2007. Classification and extraction of spatial features in urban areas using high-resolution multispectral imagery. IEEE Geoscience and Remote Sensing 
Letters 4 (2), 260-264.

Inglada, J., Christophe, E., 2009. The Orfeo Toolbox remote sensing image processing software. Dans : International Geoscience and Remote Sensing Symposium. IEEE, 12-17 July 2009, Cape Town, South Africa. pp. 733-736.

Jin, X., Davis, C., 2005. An integrated system for automatic road mapping from high-resolution multi-spectral satellite imagery by information fusion. Information Fusion 6 (4), 257 - 273.

Lacoste, C., Descombes, X., Zerubia, J., 2005. Point processes for unsupervised line network extraction in remote sensing. IEEE Transactions on Pattern Analysis and Machine Intelligence 27 (10), 1568-1579.

Laptev, I., Mayer, H., Lindeberg, T., Eckstein, W., Steger, C., Baumgartner, A., 2000. Automatic extraction of roads from aerial images based on scale space and snakes. Machine Vision and Applications 12 (1), 23-31.

Lee, H., Slatton, K., Jhee, H., 2005. Detecting forest trails occluded by dense canopies using ALSM data. Dans : International Geoscience and Remote Sensing Symposium. IEEE, 25-29 July 2005, Seoul, South Korea. Vol. 5. pp. 3587-3590.

Lugo, A., Gucinski, H., 2000. Function, effects, and management of forest roads. Forest Ecology and Management 133 (3), 249-262.

Maltamo, M., Naesset, E., Vauhkonen, J. (Eds.), 2014. Forestry Applications of Airborne Laser Scanning. Springer.

Marcantonio, M., Rocchini, D., Geri, F., Bacaro, G., Amici, V., 2013. Biodiversity, roads, and landscape fragmentation : Two mediterranean cases. Applied Geography 42, 63 - 72.

Matkan, A., Hajeb, M., Sadeghian, S., 2014. Road extraction from Lidar data using Support Vector Machine classification. Photogrammetric Engineering \& Remote Sensing, 80 (5), 249-262.

Mayer, H., Hinz, S., Bacher, U., Baltsavias, E., 2006. A test of automatic road extraction approaches. International Archives of Photogrammetry, Remote Sensing and Spatial Information Sciences 36 (Part 3), 209-214.

McRoberts, R., Tomppo, E., 2007. Remote sensing support for national forest inventories. Remote Sensing of Environment 110 (4), $412-419$.

Mena, J., 2003. State of the art on automatic road extraction for GIS update : a novel classification. Pattern Recognition Letters 24 (16), 3037-3058.

Mena, J., Malpica, J., 2005. An automatic method for road extraction in rural and semi-urban areas starting from high resolution satellite imagery. Pattern Recognition Letters 26 (9), 1201 - 1220 .

Miao, Z., Shi, W., Zhang, H., Wang, X., 2013. Road centerline extraction from high-resolution imagery based on shape features and multivariate adaptive regression splines. IEEE Geoscience and Remote Sensing Letters 10 (3), 583-587.

Montoya, J., Wegner, J., Ladicky, L., Schindler, K., 2014. Mind the gap : modeling local and global context in (road) networks. Dans : German Conference on Pattern Recognition. DAGM, 2-5 September 2014, Münster, Germany.

Poullis, C., 2014. Tensor-cuts : A simultaneous multi-type feature extractor and classifier and its application to road extraction from satellite images. ISPRS Journal of Photogrammetry and Remote Sensing 95, 93-108.

Poullis, C., You, S., 2010. Delineation and geometric modeling of road networks. ISPRS Journal of Photogrammetry and Remote Sensing 65 (2), 165-181.

Rieger, W., Kerschner, M., Reiter, T., Rottensteiner, F., 1999. Roads and buildings from laser scanner data in a forest enterprise. International Archives of Photogrammetry and Remote Sensing 32 (Part 3/W14), 185-191.

Robinson, C., Duinker, P., Beazley, K., 2010. A conceptual framework for understanding, assessing, and mitigating ecologi- cal effects of forest roads. Environmental Reviews 18, 61-86. Rochery, M., Jermyn, I., Zerubia, J., 2004. Gap closure in (road) networks using higher-order active contours. Dans : IEEE International Conference on Image Processing. IEEE, 24-27 October 2004, Singapore, Singapore. pp. 1879-1882.

Shackelford, A., Davis, C., 2003. Fully automated road network extraction from high-resolution satellite multispectral imagery. Dans : International Geoscience and Remote Sensing Symposium. IEEE, 21-25 July 2003, Toulouse, France, pp. 461463.

Soininen, A., 2014. http ://www.terrasolid.fi. Website of the Terrasolid software packages, accessed : April 30, 2014.

Unsalan, C., Sirmacek, B., 2012. Road network detection using probabilistic and graph theoretical methods. IEEE Transactions on Geoscience and Remote Sensing 50 (11), 44414453.

Vosselman, G., de Knecht, J., 1995. Road tracing by profile matching and Kalman filtering. Dans : Workshop on automatic extraction on man-made object from aerial and space images, Ascona, Switzerland. pp. 265-274.

Vosselman, G., Maas, H.-G., 2010. Airborne and Terrestrial Laser Scanning. CRC Press, Whittles Publishing, Boca Raton, FL, USA.

Wegner, J., Montoya-Zegarra, J., Schindler, K., 2013. A higherorder crf model for road network extraction. Dans : IEEE conference on Computer Vision and Pattern Recognition. IEEE, 23-28 June 2013, Portland, OR, USA.

White, R., Dietterick, B., Mastin, T., Strohman, R., 2010. Forest roads mapped using lidar in steep forested terrain. Remote Sensing 2 (4), 1120-1141.

Ziems, M., Breitkopf, U., Heipke, C., Rottensteiner, F., 2012. Multiple-model based verification of road data. ISPRS Annals of Photogrammetry, Remote Sensing and Spatial Information Sciences I-3, 329-334. 